\title{
BMJ Open Changes to postdiagnostic dementia support in England and Wales during the COVID-19 pandemic: a qualitative study
}

\author{
Alison Wheatley (D), Marie Poole, Louise Robinson (D), on behalf of the PriDem \\ study team
}

To cite: Wheatley A, Poole M, Robinson L, et al. Changes to postdiagnostic dementia support in England and Wales during the COVID-19 pandemic: a qualitative study. BMJ Open 2022;12:e059437. doi:10.1136/ bmjopen-2021-059437

- Prepublication history and additional supplemental material for this paper are available online. To view these files, please visit the journal online (http://dx.doi.org/10.1136/ bmjopen-2021-059437).

Received 19 November 2021 Accepted 11 January 2022

Check for updates

(C) Author(s) (or their employer(s)) 2022. Re-use permitted under CC BY-NC. No commercial re-use. See rights and permissions. Published by BMJ.

Population Health Sciences Institute, Faculty of Medical Sciences, Newcastle University, Newcastle upon Tyne, UK

Correspondence to Dr Alison Wheatley; alison.wheatley@ncl.ac.uk

\section{ABSTRACT}

Objectives To explore the impact of COVID-19 on postdiagnostic dementia care and support provision in England and Wales.

Design Qualitative research using semistructured interviews, via video or telephone conferencing.

Setting Services providing postdiagnostic support across health, social care and the third sector.

Participants 21 professionals previously recruited to an ongoing research programme on postdiagnostic dementia care (or colleagues, if unavailable).

Results Key themes identified from the data were: challenges caused by COVID-19; responses to those challenges, including a widespread shift to remote working; and effects of COVID-19 on future postdiagnostic support. Challenges included changing and sometimes conflicting guidelines; a lack of access to support; identifying and responding to emerging needs; emotional and physical impact of COVID-19; and balancing COVID-19 risk with other risks such as deterioration. Some dementia services closed, while others adapted and continued to provide support thus potentially widening existing inequalities. There were also some unintended positive outcomes, including improved cross-sector and multidisciplinary working between professionals.

Conclusion Delivering postdiagnostic dementia support during COVID-19 required essential adaptations. While some changes were detrimental to service users, others were identified as potentially beneficial and highly likely to become the new 'norm', for example, use of blended approaches, combining virtual and face-to-face work, thus allowing more flexible, integrated care. Our data have implications for policy and practice to improve the response to the lingering effects of COVID-19 as well as creating service provision that is more resilient to future pandemics or other periods of disruption.

\section{BACKGROUND}

Before the COVID-19 pandemic, one aim of global dementia policy was to improve postdiagnostic care and support, ${ }^{1-5}$ which is defined here as 'holistic, integrated continuing care in the context of declining function and increasing needs of family carers'. ${ }^{1}$ Guidelines in England and Wales recommend all
Strengths and limitations of this study

- This study formed part of a larger study of postdiagnostic support in England and Wales, allowing us to contextualise changes.

- Interviews were retrospective, capturing changes that had happened over time.

- Participants were geographically diverse and from a range of different health and third-sector services.

- Limitations include a relatively small sample size with limited social care input.

- Recruitment of new participants was constrained by services remaining closed or at reduced capacity during the recruitment period.

people living with dementia and their families are provided with support following diagnosis, including antidementia medications, cognitive stimulation therapy and carer psychoeducation. ${ }^{2}$ An annual dementia review in primary care is also recommended. ${ }^{6}$ However, even prior to COVID-19, considerable geographical inequalities existed in postdiagnostic support in England and Wales. ${ }^{7-9}$ COVID-19 disrupted many health and social care services, and diverting resources from non-COVID care resulted in referral and treatment backlogs ${ }^{10}$ including in dementia care. ${ }^{11}$ Such disruption had a disproportionate effect on people living with dementia. ${ }^{511-13}$ The UK went through several 'lockdowns' to reduce the spread of COVID-19 with promotion of the use of personal protective equipment (PPE), social distancing and restrictions on travel and mixing between households ${ }^{14}$; thus, services for people living with dementia were forced to suspend or adapt provision. Many care homes, in which $70 \%$ of residents have dementia, reduced social activities and stopped visitor access. ${ }^{11}$ People living with dementia were encouraged by government to 'shield' (ie, remain in their homes) as 
members of a vulnerable group, ${ }^{11}$ and there is evidence that COVID-19 mortality and morbidity disproportionately affected them. ${ }^{12}$

Several recent studies $^{15-18}$ investigated the impact of COVID-19 on people affected by dementia. They found that restrictions had negative psychological and emotional effects, ${ }^{151718}$ as well as reportedly exacerbating dementia symptoms and speeding decline. ${ }^{15-18}$ Studies of professionals providing dementia care in the UK and elsewhere $^{19-21}$ have reported challenges implementing COVID-19 restrictions, ${ }^{19-21}$ adapting to new ways of working without necessary support and training ${ }^{20}$ and balancing the well-being of professionals with provision of quality care. ${ }^{19}$ While these studies provide important insights, no study to our knowledge has explored in-depth the experiences of dementia professionals from across disciplines and sectors. The aim of this paper is to explore a range of professionals' experiences of commissioning and providing postdiagnostic dementia support in England and Wales during the COVID-19 pandemic, with a specific focus on identifying challenges and solutions.

\section{METHODS}

We used qualitative semistructured interviews to explore views and experiences of key professionals as part of an ongoing research programme (PriDem). ${ }^{22} 23$ The overall aim of PriDem was to develop a new intervention to improve postdiagnostic dementia care and support through a process of literature reviews, qualitative studies with people with dementia, carers and professionals and co-design; the present study aimed to explore changes in services during COVID-19 and ensure that the developing PriDem intervention was suitable for a post-COVID-19 care landscape.

Methods for this study were chosen for their utility in exploring experiences of professionals in relation to a specific phenomenon (ie, the COVID-19 pandemic) while making data collection as parsimonious as possible, following COVID-19 principles set out by the British Geriatrics Society. ${ }^{24}$ This led us to take a pragmatic, 'codebook thematic analysis' approach ${ }^{25}$ drawing on traditions of inductive qualitative research, enabling us to respond quickly to emerging issues while minimising burden on participants and still providing rich data.

\section{Sample and setting}

Commissioners, managers and frontline staff of services providing postdiagnostic support in England and Wales were eligible to take part. The sampling frame comprised professionals who had previously been recruited to the PriDem qualitative study before COVID-19 2627 and who had given permission for follow-up contact. If potential participants were unavailable, a colleague from the same service who had not previously been recruited to the PriDem study was eligible to take part in their place. The sample was purposive and aimed to achieve variety across sector (ie, primary care, secondary care, social care and third sector), type of service or support provided and geographical location. Sample size was restricted by availability of professionals and the time-limited nature of the project; a target sample size of 20 was selected to give scope to recruit participants with varied experiences while not placing undue burden on services.

Participants were approached for follow-up via email; contact details had either been retained with permission during the earlier phase of the PriDem study or, in the case of colleagues, were provided by potential participants who were unavailable. If there was no response after two contacts, participants were excluded.

\section{Data collection and analysis}

Data were collected between February and May 2021 by two researchers (AW and MP). A semistructured approach was chosen to provide prompts for discussion and continuity between interviews while also allowing flexibility to explore unanticipated issues raised by participants. Topic guides were used to facilitate this, informed by COVID-related subjects raised by task-group participants during PriDem intervention development. Topics covered included impacts on: services; staff; people living with dementia and carers; commissioning; and future provision (see online supplemental file 1). Interviews averaged $34 \mathrm{~min}$ and were conducted using telephone or video conferencing (Zoom or Teams), sometimes jointly with other professionals, according to participant preference. Interviews were audio recorded, professionally transcribed and checked and pseudonymised prior to analysis (AW or MP).

Data management and analysis was assisted by NVivo V.12 software. Data were analysed using framework analysis; this methodology was chosen for its utility in analysing semistructured interview transcripts and facilitating comparison and contrast. ${ }^{28}$ Both researchers familiarised themselves with the data through listening to recordings, followed by reading and rereading transcripts. Initial potential codes were then produced and refined through discussion to develop a coding frame. Ten per cent of the transcripts were dual coded (AW and MP), with agreement checked via NVivo coding comparison tools and discrepancies discussed and resolved. Remaining transcripts were single coded, and illustrative quotations were identified for each code. A series of data workshops was held to compare findings and refine final interpretations (AW, MP and LR).

\section{Reflexivity}

Two team members (AW and MP) were health services researchers with backgrounds in sociology and ethnographic methods, while one was a clinical academic and dementia specialist GP (LR); all team members had previous experience of carrying out dementia research and some also had personal experience of dementia caregiving. All team members were female. AW had previously interviewed some participants during earlier phases of the PriDem study, potentially improving rapport, while 
MP had not been involved in the earlier phases, allowing a new perspective on the data. Analysis was undertaken using an inductive approach designed to increase focus on the data and minimise the influence of the researchers, while acknowledging the inherent subjectivity of qualitative data analysis. ${ }^{25}$

\section{Ethics}

Most participants provided written consent via email or post, with verbal consent sought before interview for those who had not returned consent forms. Participants are identified in this paper only via pseudonym.

\section{RESULTS}

Eighteen interviews were conducted with 21 professionals: six commissioners, eight service managers or team leads and seven frontline staff. Professionals worked in primary care (6), secondary care (9), joint health and social care (1) and third sector (5). Services included memory assessment services, community dementia teams and specialist support services for black, Asian and minority ethnic (BAME) people and people with young onset dementia. Professional backgrounds of participants included general practitioner (GP), dementia specialist nurse, occupational therapist (OT) and dementia support worker. The geographical spread of the sample was across the following UK National Health Service (NHS) regions: London (5); Midlands (1); North East and Yorkshire (5); North West (2); South East (3); South West (2); and Wales (3).

Findings are organised into three themes, as shown in table 1 and discussed in detail in the following sections.

\section{Challenges for postdiagnostic support}

A number of challenges for postdiagnostic support emerged during COVID-19, and services were forced to come up with creative ways of overcoming them, involving a high degree of trial and error.

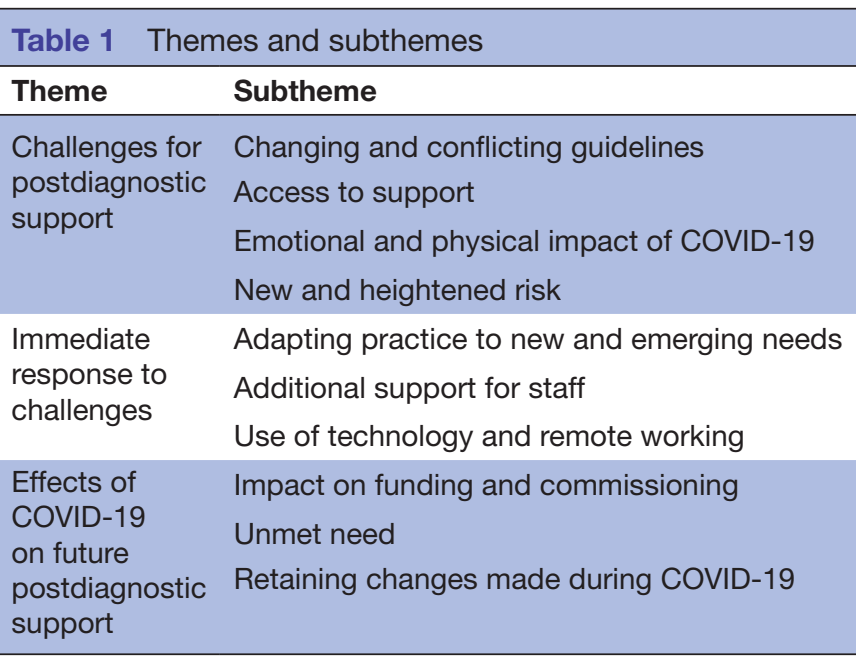

Changing and conflicting guidelines

Understanding and responding to guidelines from various sources, including national and devolved government, local councils and NHS organisations, could be challenging. Inconsistencies created particular difficulties for services involving workers from more than one sector. Staff described this period as 'stop-start' due to ongoing uncertainty.

Moreover, guidelines were not always person centred or appropriate for people living with dementia. Language such as 'lockdowns' and 'social distancing' led to some social support services being unnecessarily cautious, as the boundaries of 'social activity' and 'support' could be ill-defined or misinterpreted:

[Permission] for support groups of up to 15 people has been there for quite a while now, at least since the autumn, possibly longer ago than that. But a lot of things that our community groups put on for people with dementia, just have a very, sort of, human, social feel [...] [so group facilitators] tend to interpret what they can and can't do with those groups and activities according to the regulations that govern our social lives. [...] I think the fact that people talked about the second lockdown and the third lockdown doesn't help. [...] I've had to say, 'you are health and care and support. I know it's got the feel of a social gathering and that's why it's nice for people because it feels like a social gathering, but this is doing people good'. (S016, joint commissioner)

PPE could be confusing or frightening for people living with dementia, as well as hindering communication through hiding mouth movement and facial expressions. Some service users preferred remote contact to visits with full PPE:

[Carers] said, 'There's no point you coming with your PPE because [the person living with dementia] finds it really stressful.' I've tried the see-through ones [facemasks], but you have to still wear your visor. [...] you've got your aprons and you've got your gloves, all that, and it's still, you end up still traumatising the patient. (S057, community dementia support team)

Some professionals therefore suggested that policies mandating PPE for face-to-face visits should have been flexible, especially where physical contact was unnecessary.

\section{Access to support}

The reduced availability of staff, either due to furloughs, redeployment to COVID-19 care, or their status as clinically vulnerable, additionally affected whether services could operate. While some dementia services were able to adapt to guidelines and resource challenges, others were forced to close entirely at various times, including some memory assessment services. Services providing group social interaction and meaningful activity were particularly vulnerable to closure due to a lack of space to implement social distancing. Closures placed extra pressure 
on other services, including crises arising from reduced social contact and support networks; problems providing support where people with suspected dementia had not yet received a diagnosis; reduced access to support and advice for staff in other sectors; and inappropriate referrals to dementia services:

[The dementia service has] had two referrals for hands [...] it's [an] OT problem to look at the contracture, and a nursing problem to look at the [hand] hygiene. But it's all come to us, hasn't it, and it's strange $[\ldots]$ how we've become seen as a Jack of All Trades. (S056, community dementia support team)

Transitions for people living with dementia living in the community needing admission to hospital or a care home, either permanently or for respite, were also affected. Reduced or no visiting to relatives living in care homes was identified by professionals as a major impact of COVID-19.

\section{Emotional and physical impact of COVID-19}

Working in dementia care during COVID-19 affected professionals both emotionally and physically. Many staff worked longer hours than usual and some felt that they were approaching 'burnout'. In addition to personal experiences with COVID-19, facing more patient deaths than usual and addressing the heightened emotional support needs of service users was challenging. Staff also reported feelings of helplessness when balancing precaution with action:

[...] historically we would have just said, 'Right, this person needs an emergency visit,' we go out and we try to put as much in place as we can. However, if that person has got COVID then we cannot just go rushing out because then we are putting all our colleagues and other people at risk. So we feel quite disabled. (S603, dementia support worker)

Working from home reduced professionals' access to informal peer support such as debriefs with colleagues following emotional telephone consultations. Moreover, lack of travel time between 'visits' either at home or on site reduced opportunities for reflexive practice and lead to increased pressure:

[...] because we're continuously on the phones, there's no breaks [...] we've increased our contacts, but I think at the detriment of our health and wellbeing because we don't have any time to just offload. Breathe, get coffee and actually offload. (S057, community dementia support team)

\section{New and heightened risk}

Managing risk was challenging for services and service users alike. Recognising and treating the symptoms of COVID-19 in people living with dementia were identified as specific areas for improvement. However, while managing COVID-19 was important, it was also necessary to balance other heightened risks for people living with dementia, including increased isolation and worsening symptoms. Professionals reported that many families had paused in-home services, such as paid home care, because of COVID-19 risk. One GP practice had put every patient with dementia on the NHS 'shielding list', which allowed them to gain priority access to some designated services:

[...] we made a decision in the practice a year ago, to put all of our patients with dementia on the shielding list. The rationale being that, yes, they were more vulnerable, but also, it would be easier for them to access the support services that were available. (S043, GP commissioner)

However, others reported that defining people living with dementia as vulnerable led to overcautiousness with some missing out on beneficial interventions such as outside exercising, arguing that providing better information could have reduced anxiety and encouraged engagement in activities.

\section{Immediate response to challenges}

While responses varied widely, there were several examples of good practice.

\section{Adapting practice to new and emerging needs}

Innovative adaptations that allowed services to remain open included social distancing indoors, meeting outdoors and reallocating existing resources, such as assigning volunteers to telephone helplines or one-to-one support rather than group support activities:

[We had] peer supporters phoning up people living with dementia and their carers and saying, 'Can we have a cup of tea outside your door?' You know, 'Can we meet to just have a check in, see how things are, what you need support with, how we can help you in terms of caring?' [...] because people were worried and frightened about catching coronavirus, and a lot of the carers were struggling to come out of the house. (S037, NHS commissioner)

Service users' needs were often different than usual, including increased emotional support needs due to isolation, and varied across the different phases of lockdown, requiring reactiveness in everyday practice. In some services, prebooked appointments were replaced with telephone hotlines, often focusing on hospital admissions avoidance and crisis management. However, this type of working was more challenging for staff in terms of managing time and workload. Several services combined reactive approaches with proactive welfare checks for those with the highest levels of need. Reviewing and triaging caseloads at the beginning of lockdown enabled services to prioritise and identify matters that could have been missed:

We rated them [service users] either red, amber or green, from just our knowledge of their social set up, their care needs and their severity of symptoms [...] 
you'd stumble across that they hadn't been put on a list for getting groceries, they didn't have anyone who could do shopping for them, so we'd kind of have to try and get that sorted and signposted one of the charities or someone who could help. We came across a couple of safeguarding [elder abuse and neglect] things. (S046, GP)

\section{Additional support for staff}

Some managers put additional support in place for staff, including formal check-ins, regular breaks and access to counselling, with some prioritising managing staff wellbeing over other duties:

I have very much stepped back from patient care and I have just been here to support the staff, because the staff and just needing that extra support like I have never known in my career, and I have managed staff for a long time. (S036, memory service manager)

This additional support was valued by frontline professionals.

Cross-sector advice and support for other professionals were also important. One memory service with a wellestablished care home liaison team adopted a proactive approach to support care home staff:

[...] our care home liaison staff were setting up regular touch-bases with the care home teams, in many ways kind of offering a bit of long-arm supervision. They were troubleshooting over the phone, but often times it was just care teams phoning up just to say, 'Can we talk through stuff?' wanting someone outside the building to understand what their pressures were and what was going on for them. (S214, memory service manager)

\section{Use of technology and remote working}

Perhaps the most widespread solution to COVID-19 restrictions was technology and remote working. The first response was frequently to implement additional telephone support, as people living with dementia were thought more likely to have access to telephones compared with computers, tablets or smartphones. However, webbased solutions were also implemented. Diverse services including case management, diagnostic services and support groups were able to continue remotely, which had benefits and drawbacks for both service users and providers.

\section{Benefits and drawbacks of remote consultation for service users}

Potential benefits to remote consultations included increased convenience, comfort and control for people living with dementia and carers:

A carer yesterday said to me she finds it easier to engage, expressing herself about quite difficult and emotional issues, on the screen [...] If you're not finding it helpful or you want to disengage, you're not going to be faced with social awkwardness thinking 'how do I extract myself now and leave?' You can make an excuse and log off. (S034, team lead, young onset dementia service)

Virtual methods allowed services to extend their reach, enabling the inclusion of carers who lived at distance or had other commitments that reduced their capacity to attend face-to-face meetings, as well as more service users than they ordinarily would meet due to reductions in travel time:

[...] you might be able to make 8 to 10 calls during the day, whereas visits, you might only do 3 to 4 visits during the day. So, it's definitely being able to reach out more responsively, I would say, and quicker to people, and having access, which has been a positive. (S015, dementia specialist nurse)

Several services that usually provided home visits implemented a 'triage' process to assess which visits were in service users' best interests and should take place and which should be carried out remotely. Some elements of support, including cognitive assessments, were difficult to achieve successfully remotely, and others, such as diagnosis disclosure or planning for end of life, could be unsuitable because of their emotional nature, particularly for people living alone. Remote support also reduced access to non-verbal cues; professionals felt they did not have the 'full picture' of what was going on in the life of the person, despite being able to see them on the screen:

[...] you don't always pick up the subtleties of what somebody is perhaps not telling you, or the environment that they are living in. If you can't see all of that you only get part of a picture. (S049, dementia specialist nurse)

There were also concerns around accessibility, especially for those who did not have a carer to help them. Potential problems with telephones included reluctance to answer unknown numbers and lack of assistive technology. Dementia symptoms, such as difficulties with memory and concentration, compounded by loss of contextual information, eye contact and body language, could make remote consultations and meetings confusing and tiring. Some professionals were therefore concerned that people living with dementia's voices were marginalised when interacting remotely.

Some families were supported by community-based initiatives that provided assistive technology for telephone calls, loaned hardware such as computer tablets and/or supported users to download apps and connect to meetings; areas where such initiatives had already been in place prepandemic were better placed to adapt. Other types of support included telephone coaching in advance of sessions and using a static joining link to reduce complexity. Professionals highlighted that many people have also gained confidence and technological skills in informal ways during lockdown: 
[...] whereas Nan would have been reluctant to have a video call ever, [now] because that's the only way she can see her grandchildren, she's trying it and actually become more confident with it. So, when you are offering these interventions in different ways, people are much more open to it and the confidence is building. ( $\mathrm{S} 054$, memory service manager)

\section{Benefits and drawbacks of remote working for staff}

Virtual solutions adapted rapidly along with changing needs and guidelines. Different videoconferencing applications were considered more appropriate for different types of interactions, for example, Zoom for group activity, Attend Anywhere for individual consultations and Teams for interaction between professionals; this was usually for reasons of accessibility, usability and security, requiring staff to become proficient in several platforms. There were additional logistical challenges for services that had not previously had infrastructure in place, for example, obtaining computers, setting up remote workspaces or problems arising from the need to use personal equipment for work.

In addition to patient-facing work, professionals used videoconferencing to support cross-service and crosssector working, facilitating multidisciplinary team meetings, care home ward rounds and networking and training events:

These meetings were not happening before, or they used to happen once a month in a boardroom somewhere. If you trudged over there and spent hours finding parking before you got into the meeting, all that kind of nonsense which sort of beat the purpose. That sort of thing [meeting] is happening far more now. I am far more aware of who my counterparts in local authority are. So, if I need to raise questions or get answers I know where to go. (S059, GP commissioner)

However, working remotely could reduce informal networking opportunities.

\section{Effects of COVID-19 on future postdiagnostic support}

Participants thought the negative effects of COVID-19 on dementia care were likely to be ongoing and long lasting. However, many professionals also felt there were positive changes that could be integrated into future practice.

\section{Impact on funding and commissioning}

COVID-19 had a financial effect on dementia services; NHS funds had been diverted to efforts to manage COVID19 , and local authority and third-sector funds were also negatively affected. However, some additional funding streams became available, such as those to support digital participation or services set up to support people during COVID-19. Commissioners thought that policy decisions, such as priority being given to COVID-19 recovery and vaccine programmes, may have ongoing implications for postdiagnostic support:
I think the changing politics and the changing environment within the CCG (NHS Clinical Commissioning Group) and new prime minister and all the rest of it, new priorities, has made a difference [...] I've lost people who were up to speed with what I was doing. I feel as I'm having to start all over again. (S043, GP commissioner)

\section{Unmet need}

With the reduction in service provision, professionals expressed concern about unmet dementia support needs. Those attending services once they reopened were found to be more advanced in their dementia due to a combination of delays in diagnosis, lack of proactive support and the effects of isolation:

[...] my bigger worry is, is just how much unmet need is going to start to emerge as we're coming out of lockdown $[\ldots]$ we're starting to see now people presenting at much more crisis points because they've been kind of rumbling along for a long time without seeking help, so I suspect it's actually going to be much worse in the coming year. (S050, GP commissioner)

Where resource for dementia services had been reprioritised to COVID-19 responses, there were concerns that this had not yet been assigned back to dementia services. There was also concern over the lasting effects of COVID-19 and long COVID, particularly exacerbation of symptoms in people with existing dementia or potential new forms of memory loss. As these issues were emerging and not fully understood, this was an example of the need for adaptability and responsiveness to ongoing change during COVID-19.

\section{Retaining changes made during COVID-19}

Several professionals reported that COVID-19 had acted as a catalyst for innovation and changes in attitudes, including of some commissioners and providers of dementia support. Barriers such as reluctance to provide remote services were reduced, enabling changes that had previously been considered impossible:

I think there were some very-what's the word?some sort of sacred cows [among clinicians in dementia services] [...] A bit of, 'We can't possibly do it in that way,' and then, suddenly, they had to. Then, suddenly, they think, 'Actually, that has worked quite well.' So, in some ways, it has been a bit of an engine for innovation. (S708, NHS commissioner)

Professionals from all sectors identified changes they wished to retain, particularly 'blended' approaches to remote working that would enable people living with dementia and/or carers to choose in-person or virtual meetings:

If people [in the group] still want to keep music sessions online and meet [face-to-face] on Thursdays, because our music sessions are on Mondays, then we 
will probably just keep those virtual. But depending on what the client is going to want, really. I can't decide on that, so it will be up to them. (S047, BAME dementia service manager)

While some staff expressed concerns and hesitancy around blended working, these were mainly concerns around online provision and suitability of platforms for providing dementia support. Other changes that service managers felt could be taken forward included the idea of triaging their caseloads to identify needs of people living with dementia and carers and plan future contacts.

\section{DISCUSSION}

This paper reports an in-depth qualitative study to describe the experiences of a wide range of professionals-service commissioners, managers and frontline staff-providing postdiagnostic dementia care in England and Wales during COVID-19. A number of challenges arose, necessitating adaptations to services including reductions in face-to-face work and widespread adoption of technology. Many of these changes were refined through 'trial and error', with services required to be creative and resourceful. While some changes were detrimental to service users, others were potentially beneficial, with staff feeling these may become the 'new norm'. Chief among these was a blended approach involving both remote and face-to-face work, allowing flexible, convenient and tailored care, with improved choice and enhanced opportunities for carer involvement. Additional benefits to services and staff included improved professional development opportunities and better integration between services and sectors. Other examples of good practice included triaging caseloads and attending to professionals' emotional and physical support needs.

The challenges identified were consistent with other studies of professionals from different contexts, including ambiguity around guidelines,${ }^{20}{ }^{21}$ the difficulty of balancing COVID-19 risk and restrictions with good quality care ${ }^{19} 20$ and the emotional impact of COVID-19 on professionals. ${ }^{19}{ }^{20}$ However, while some of the challenges we identified were unique to COVID-19, others were exacerbations of existing barriers to good postdiagnostic support, including poorly managed cross-sector working and care transitions, inequalities in access to specialist support and limited anticipation and planning for future needs. ${ }^{8}$ Services where existing challenges had been addressed pre-COVID-19 experienced fewer disruptions. Ongoing challenges included unmet need in people living with dementia ${ }^{29}$; participants expected that these effects would be felt for many months to come, even once restrictions had been lifted. This is congruent with other research suggesting that COVID-19 and resulting isolation had both increased emotional support needs ${ }^{15} 1718$ and worsened dementia symptoms. ${ }^{15-18}$

Our results add to the evidence that older people and many people living with dementia can use technology, ${ }^{30} 31$ as improvements in the ability and confidence of both staff and service users in the use of technology is a potential ongoing effect of the response to COVID-19. Consistent with the findings of a recent review, ${ }^{32}$ facilitators for virtual working included support from a carer and/or group organiser and accessibility of technology. However, professionals identified some specific challenges to using technology with some service users, echoing findings from people living with dementia and carers. ${ }^{15} \mathrm{~A}$ blended approach, rather than a wholesale shift to remote working, was therefore seen as the most likely result for future postdiagnostic support. ${ }^{20}$ Blended working would enable services to work more flexibly and responsively to the needs of service users and provide opportunities for more people to engage; however, the risk of marginalising the voices of people living with dementia should be considered when deciding which approach is suitable and acceptable to them, especially where there are conflicting preferences between people living with dementia and carers. In order to facilitate blended approaches and help mitigate remaining challenges, including challenges specific to dementia, continuing training in digital literacy should be incorporated into existing recommendations about education and support for people living with dementia and carers, ${ }^{2}$ as well as the development of dementia-friendly approaches to technology. ${ }^{31}$

\section{Strengths and limitations}

One strength of this study was that it formed part of a larger study of postdiagnostic support in England and Wales, allowing us to contextualise changes. Interviews were retrospective, capturing changes that had happened over time. Participants were geographically diverse and from a range of different health and third-sector services. Limitations include a relatively small sample size with limited social care input. However, this reflects the small number of social care services included in the sample from the original study ${ }^{26}$; recruitment of new participants was constrained by services remaining closed or at reduced capacity during the recruitment period.

\section{Implications for practice and policy}

Our data demonstrate that positive lessons can be learnt to improve the response to the lingering effects of COVID-19 and create service provision that is more resilient to future pandemics or other periods of disruption. First, addressing existing challenges to postdiagnostic support, such as integration between services and sectors, could help services and professionals better cope with crises and reduce inequalities of provision. Second, services that continued to function during COVID-19 learnt to manage persistent uncertainty by generating and embracing creative solutions, including rapid uptake of new technologies, leading to benefits for both service providers and users. Better support and training are still needed to enable professionals, people living with dementia and carers to make best use of technology within usual care; the increased uptake of remote 
working in this period has prepared the groundwork for blended working approaches to become the 'new norm', although it is important to ensure that this is appropriate for people living with dementia. Finally, it is vital that future pandemic policy and healthcare action plans adopt a more person-centred and flexible approach to risk management for subgroups of the population like people living with dementia where the risks of COVID-19 infection should be balanced with other risks such as isolation, as with other aspects of dementia care and support.

Twitter Alison Wheatley @AlisonLWheatley, Marie Poole @mariepoole77 and on behalf of the PriDem study team @PriDemProject

Acknowledgements Administrative support was provided by Angela Mattison. We would like to thank all participants for their support and involvement in the study. A conference abstract related to this work was previously published.

Collaborators The PriDem study team Louise Robinson (Newcastle University), Alison Wheatley (Newcastle University), Louise Allan (University of Exeter), Claire Bamford (Newcastle University), Sube Banerjee (University of Plymouth), Greta Brunskill (Newcastle University), Alistair Burns (NHS England and NHS Improvement), Sophie Dimitriadis (International Longevity Centre), Karen Harrison Dening (Dementia UK), Sarah Griffiths (University College London), Derek King (London School of Economics), Martin Knapp (London School of Economics), Doug Lewins (PPI co-applicant), Jill Manthorpe (King's College London), Greta Rait (University College London), Emily Spencer (University College London), Sue Tucker (PPI co-applicant), Kate Walters (University College London), Jane Wilcock (University College London) and Raphael Wittenberg (London School of Economics)

Contributors AW, MP and LR designed the study and drafted the manuscript. AW and MP collected and analysed the qualitative data. Members of the PriDem study team and all authors revised and approved the final manuscript. LR is the guarantor of the study.

Funding This work was supported by funding from Alzheimer's Society Centre of Excellence grant number 331.

Disclaimer The funder played no role in data collection, interpretation or reporting

Competing interests None declared.

Patient and public involvement statement A multi-stakeholder Dementia Care Community (DCC) group was established as part of the wider PriDem programme to inform all stages of the research, involving people with dementia, their family members, and health and social care professionals from a range of organisations. The PriDem study project team includes two PPI co-applicants (DL and ST) who were involved in the management of the programme.

Patient consent for publication Not applicable.

Ethics approval Approval was granted by NHS Research Ethics Committee Wales 3 (reference 18/WA/0349). Participants gave informed consent to participate in the study before taking part.

Provenance and peer review Not commissioned; externally peer reviewed.

Data availability statement Data are available on reasonable request. Further information available at https://doi.org/10.25405/data.ncl.c.5718116.v2

Supplemental material This content has been supplied by the author(s). It has not been vetted by BMJ Publishing Group Limited (BMJ) and may not have been peer-reviewed. Any opinions or recommendations discussed are solely those of the author(s) and are not endorsed by BMJ. BMJ disclaims all liability and responsibility arising from any reliance placed on the content. Where the content includes any translated material, BMJ does not warrant the accuracy and reliability of the translations (including but not limited to local regulations, clinical guidelines, terminology, drug names and drug dosages), and is not responsible for any error and/or omissions arising from translation and adaptation or otherwise.

Open access This is an open access article distributed in accordance with the Creative Commons Attribution Non Commercial (CC BY-NC 4.0) license, which permits others to distribute, remix, adapt, build upon this work non-commercially, and license their derivative works on different terms, provided the original work is properly cited, appropriate credit is given, any changes made indicated, and the use is non-commercial. See: http://creativecommons.org/licenses/by-nc/4.0/.
ORCID iDs

Alison Wheatley http://orcid.org/0000-0002-6051-0286

Louise Robinson http://orcid.org/0000-0003-0209-2503

\section{REFERENCES}

1 Alzheimer's Disease International. World Alzheimer report 2016. Improving healthcare for people with dementia. London: Alzheimer's disease international, 2016. https://www.alz.co.uk/research/worldreport-2016

2 National Institute for Health and Care Excellence. Dementia: assessment, management and support for people living with dementia and their carers (NG97). 201820 June. Contract No.: NG97.

3 NHS England. The well pathway for dementia, 2016. Available: https://www.england.nhs.uk/mentalhealth/wp-content/uploads/sites/ 29/2016/03/dementia-well-pathway.pdf

4 World Health Organisation. Global action plan on the public health response to dementia 2017-2025. Geneva: World Health Organisation, 2017. https://apps.who.int/iris/bitstream/handle/10665/ 259615/9789241513487-eng.pdf?sequence=1

5 Alzheimer's Disease International.. From plan to impact IV: progress towards targets of the WHO global action plan on dementia. London: Alzheimer's Disease International, 2021.

6 NHS England and NHS Improvement. 2020/21 general medical services (GMS) contract quality and outcomes framework (QOF), 2020. Available: https://www.england.nhs.uk/wp-content/uploads/ 2020/09/C0713-202021-General-Medical-Services-GMS-contractQuality-and-Outcomes-Framework-QOF-Guidance.pdf

7 Frost R, Walters K, Wilcock J, et al. Mapping post-diagnostic dementia care in England: an e-survey. J Integr Care 2020;29:22-36.

8 Wheatley A, Bamford C, Brunskill G, et al. Implementing postdiagnostic support for people living with dementia in England: a qualitative study of barriers and strategies used to address these in practice. Age Ageing 2021;50:2230-7.

9 Alzheimer's Society. From diagnosis to end of life: the lived experiences of dementia care and support. Alzheimer's Society and the Peter Sowerby Foundation, 2020.

10 British Medical Association. The hidden impact of COVID-19 on patient care in the NHS in England. London: British Medical Association, 2020. https://www.bma.org.uk/media/2840/the-hiddenimpact-of-covid_web-pdf.pdf

11 Alzheimer's Society. Worst hit: dementia during coronavirus. Alzheimer's Society, 2020.

12 Burns A, Howard R. COVID-19 and dementia: a deadly combination. Int J Geriatr Psychiatry 2021;36:1120-1.

13 Liu KY, Howard R, Banerjee S, et al. Dementia wellbeing and COVID-19: review and expert consensus on current research and knowledge gaps. Int J Geriatr Psychiatry 2021;36:1597-639.

14 Institute for Government. Timeline of UK coronavirus lockdowns, March 2020 to March 2021. London: Institute for Government, 2021. https://www.instituteforgovernment.org.uk/sites/default/files/ timeline-lockdown-web.pdf

15 Tuijt R, Frost R, Wilcock J, et al. Life under lockdown and social restrictions - the experiences of people living with dementia and their carers during the COVID-19 pandemic in England. BMC Geriatr 2021;21:301.

16 Giebel C, Cannon J, Hanna K, et al. Impact of COVID-19 related social support service closures on people with dementia and unpaid carers: a qualitative study. Aging Ment Health 2021;25:1-8.

17 Talbot CV, Briggs P. 'Getting back to normality seems as big of a step as going into lockdown': the impact of the COVID-19 pandemic on people with early to middle stage dementia. Age Ageing 2021;50:657-63.

18 Tsapanou A, Papatriantafyllou JD, Yiannopoulou K, et al. The impact of COVID-19 pandemic on people with mild cognitive impairment/dementia and on their caregivers. Int J Geriatr Psychiatry 2021;36:583-7.

19 RHM L, Shalhoub R, Sridharan BK. The experiences of the community pharmacy team in supporting people with dementia and family carers with medication management during the COVID-19 pandemic. Res Soc Admin Pharm 2021;17:1825-31.

20 Bhome R, Huntley J, Dalton-Locke C, et al. Impact of the COVID-19 pandemic on older adults mental health services: a mixed methods study. Int J Geriatr Psychiatry 2021;36:1748-58.

21 Banerjee D, Vajawat B, Varshney P, et al. Perceptions, experiences, and challenges of physicians involved in dementia care during the COVID-19 Lockdown in India: a qualitative study. Front Psychiatry 2020;11:615758. 
22 PriDem Study Project Team. Primary care-led post diagnostic dementia care (PriDem): developing evidence-based, person-centred sustainable models for future care, 2021. Available: https://research. ncl.ac.uk/pridem/

23 Wheatley A, Poole M, Robinson L. 211 - Changes to post-diagnostic dementia support in England and Wales during the COVID-19 pandemic. Int Psychogeriatr 2021;33:13.

24 British Geriatrics Society. BGS statement on research for older people during the COVID-19 pandemic 2020. Available: https://www. bgs.org.uk/resources/covid-19-bgs-statement-on-research-for-olderpeople-during-the-covid-19-pandemic

25 Braun V, Clarke V. Conceptual and design thinking for thematic analysis. Qual Psychol 2021.

26 Wheatley A, Bamford C, Brunskill G, et al. Task-shifted approaches to postdiagnostic dementia support: a qualitative study exploring professional views and experiences. BMJ Open 2020;10:e040348.
27 Bamford C, Wheatley A, Brunskill G, et al. Key components of post-diagnostic support for people with dementia and their carers: a qualitative study. PLoS One 2021;16:e0260506.

28 Gale NK, Heath G, Cameron E, et al. Using the framework method for the analysis of qualitative data in multi-disciplinary health research. BMC Med Res Methodol 2013;13:1-8.

29 Miralles O, Sanchez-Rodriguez D, Marco E, et al. Unmet needs, health policies, and actions during the COVID-19 pandemic: a report from six European countries. Eur Geriatr Med 2021;12:193-204.

30 Poole M, Davis N, Robinson L. Massive open online courses: enhancing caregiver education and support about dementia care towards and at end of life. Age Ageing 2020;49:171-4.

31 Di Lorito C, Duff C, Rogers C, et al. Tele-rehabilitation for people with dementia during the COVID-19 pandemic: a case-study from England. Int J Environ Res Public Health 2021;18 doi:10.3390/ ijerph18041717

32 Dowson B, Schneider J. Online singing groups for people with dementia: scoping review. Public Health 2021;194:196-201. 